\title{
Realização de Angioplastia Coronária com Volume Total de Três Mililitros de Contraste
}

\author{
Gustavo Monteiro', Rodolfo Staico', Wersley Araújo', Ricardo Costa ${ }^{1}$, Áurea Chaves ${ }^{1}$, Fausto Feres ${ }^{1}$
}

\section{RESUMO}

A administração sistêmica de meio de contraste iodado pode ocasionar o desenvolvimento de nefropatia induzida por contraste, cuja incidência é consideravelmente maior nos diabéticos e idosos e naqueles com doença renal preexistente, e está vinculada a aumento da morbidade e da mortalidade. Não há tratamento específico para a nefropatia induzida por contraste e uma das maneiras de preveni-la é usar pequeno volume de contraste. Relatamos um caso de implante de stent coronário em paciente com insuficiência renal crônica e síndrome coronária aguda, no qual foram utilizados apenas $3 \mathrm{ml}$ de contraste, empregando o sistema de injeção $\mathrm{ACIST}^{\mathrm{TM}}$ e o ultrassom intracoronário para auxiliar o procedimento.

DESCRITORES: Angioplastia. Stents. Insuficiência renal crônica. Síndrome coronária aguda. Meios de contraste.

A administração de meios de contraste iodado em exames diagnósticos e terapêuticos pode ocasionar o desenvolvimento de insuficiência renal aguda, denominada nefropatia induzida por contraste, e definida como aumento $>25 \%$ da creatinina sérica em relação aos valores pré-procedimento, 72 horas após a administração do contraste. Agentes de contraste radiológico são a terceira causa mais comum de nefropatia entre os pacientes internados, sendo responsáveis por $11 \%$ a $12 \%$ dos casos. A nefropatia induzida por contraste tem incidência consideravelmente maior nos diabéticos, nos idosos e naqueles com doença renal preexistente, quando comparados com a população geral. Está vinculada a consequências como hospitalização prolongada, diálise e mortalidade. ${ }^{1-3}$ Não há tratamento específico e o manejo da doença visa à correção de distúrbios hidroeletrolíticos

1 Instituto Dante Pazzanese de Cardiologia - São Paulo, SP, Brasil. Correspondência: Rodolfo Staico. Av. Dr. Dante Pazzanese, 500 Vila Mariana - São Paulo, SP, Brasil - CEP 04012-909 E-mail: rstaico@hotmail.com

Recebido em: 26/9/2011 • Aceito em: 5/12/2011

\section{ABSTRACT}

Coronary Angioplasty Performed with a Total Volume of Three Milliliters of Contrast

The systemic administration of iodinated contrast media may lead to the development of contrast-induced nephropathy, with a particular higher incidence in diabetics, in the elderly and in those with preexisting renal disease, and is associated with increased morbidity and mortality. There is no specific treatment for contrast-induced nephropathy and one way to prevent it is to use a small volume of contrast media. We report a case of coronary stent implantation in a patient with chronic renal failure and acute coronary syndrome, using only $3 \mathrm{ml}$ of contrast, utilizing the ACIST ${ }^{\mathrm{TM}}$ injection system and intravascular ultrasound to aid the procedure.

KEY-WORDS: Angioplasty. Stents. Renal insufficiency, chronic. Acute coronary syndrome. Contrast media.

e manutenção da volemia. A melhor terapêutica é sua prevenção, que pode ser realizada tomando-se algumas precauções ${ }^{1-4}$ :

- nos casos de disfunção renal prévia (clearance de creatinina < $60 \mathrm{ml} / \mathrm{min}$ ), é necessário "preparo renal" com a administração de soro fisiológico 0,9\% intravenoso, 6-12 horas antes e 12-24 horas pós-procedimento a hidratação adequada é a única estratégia profilática benéfica para a nefropatia induzida por contraste, com robusta e inconteste evidência científica na atualidade;

- usar volume pequeno de contraste (nos casos com clearance de creatinina $<60 \mathrm{ml} / \mathrm{min}$, usar agente de contraste de baixa osmolaridade ou iso-osmolar) a razão do volume de contraste/clearance de creatinina $>$ 2,62 é um preditor independente de nefropatia induzida por contraste em pacientes não-selecionados, após intervenção coronária percutânea $(\mathrm{ICP})^{5}$;

- evitar a depleção de volume e o uso concomitante de fármacos anti-inflamatórios;

- evitar a readministração do meio de contraste dentro de 48-72 horas do exame índex (ou dentro de 7-10 dias, no caso de nefropatia induzida por contraste); 
- a injeção manual de contraste com seringa é o método atual mais utilizado para a realização de angiografia coronária; porém, o sistema de injeção ACIST ${ }^{\mathrm{TM}}$ (ACIST Medical Systems, Eden Prairie, Estados Unidos) permite injeções controladas de contraste, é de uso fácil e oferece imagens com qualidade satisfatória, mesmo com cateteres de pequeno calibre. O painel de controle do equipamento permite configurar os parâmetros de cada injeção, ajustando o volume e o fluxo de contraste e reduzindo, ao final, a quantidade de contraste utilizada nos procedimentos. ${ }^{6-8}$

\section{RELATO DO CASO}

Paciente do sexo masculino, 77 anos, portador de hipertensão arterial sistêmica, dislipidemia, insuficiência renal crônica não-dialítica (creatinina sérica $=$ $1,3 \mathrm{mg} / \mathrm{dl}$, clearance de creatinina calculado $=35 \mathrm{ml} / \mathrm{min}$ ), psoríase e neoplasia de reto, tratada com cirurgia, quimioterapia e radioterapia há três anos. Estava em uso regular de atenolol (25 mg/dia), enalapril (10 mg/dia), ácido acetilsalicílico (100 mg/dia) e sinvastatina (20 mg/dia).

Apresentou infarto agudo do miocárdio sem supradesnivelamento do segmento ST, sendo internado na Unidade Coronária (UCO) do Instituto Dante Pazzanese de Cardiologia em setembro de 2011 e medicado adicionalmente com clopidogrel $(300 \mathrm{mg}$ de ataque e $75 \mathrm{mg} / \mathrm{dia}$ ) e enoxaparina ( $1 \mathrm{mg} / \mathrm{kg} / \mathrm{dia})$. Evoluiu assintomático e foi submetido a cinecoronariografia, após "preparo renal", no terceiro dia de internação. O exame mostrou: circulação coronária de padrão balanceado; coronária direita, tronco da coronária esquerda e artéria descendente anterior sem lesões obstrutivas significativas; e artéria circunflexa com lesão obstrutiva de $30 \%$ no óstio, lesão de $70 \%$ proximal seguida por outra de $90 \%$ no primeiro ramo marginal, o qual exibe lesão de $60 \%$ no óstio de um sub-ramo (Figura 1). A ventriculografia esquerda evidenciou volumes finais preservados e hipocinesias apical e lateral discretas.
Foram utilizados, no exame, $40 \mathrm{ml}$ de contraste de baixa osmolaridade Hexabrix ${ }^{\mathrm{TM}}$ (Guerbet LLC, Bloomington, Estados Unidos).

Após a cinecoronariografia, o paciente retornou para a UCO e foi decidida revascularização miocárdica percutânea com implante de stent na lesão proximal da circunflexa, programada para duas semanas, em decorrência de insuficiência renal prévia. Após dois dias, o paciente apresentou instabilização do quadro, com angina de repouso refratária ao tratamento clínico. A creatinina sérica mantinha-se em 1,3 mg/dl. Decidiu-se pela realização de angioplastia coronária com mínima quantidade de meio de contraste de baixa osmolaridade, com auxílio do sistema de injeção ACIST $^{\text {TM }}$ CMS2000 e do ultrassom intracoronário (sistema iLab ${ }^{T M}$ e cateter Atlantis ${ }^{\mathrm{TM}}$ SR Pro, Boston Scientific, Natick, Estados Unidos) para guiar o implante do stent.

Durante o procedimento terapêutico, a cateterização da coronária esquerda com o cateter-guia Launcher ${ }^{\mathrm{TM}}$ EBU 3,5/6 F (Medtronic, Minneapolis, Estados Unidos), a passagem do fio-guia 0,014 polegada CholCE PT2 ${ }^{\text {TM }}$ MS (Boston Scientific, Natick, Estados Unidos) e o posicionamento do balão TREK ${ }^{\mathrm{TM}}$ 2,5/20 mm (Abbott Vascular, Temecula, Estados Unidos) para a pré-dilatação da lesão proximal da circunflexa foram realizados sem o uso de contraste, e orientados apenas pela anatomia visualizada na cinecoronariografia prévia. Após a pré-dilatação até 13 atm e desinsuflação do balão, mas mantendo-o ainda na mesma posição para mensuração da extensão da lesão por meio de suas marcas radiopacas, foi injetado $1 \mathrm{ml}$ de contraste para controle angiográfico, visando à constatação do bom fluxo coronário e da ausência de dissecção que alterasse o planejamento inicial. Foi, então, realizado ultrassom intracoronário para determinar a extensão e o diâmetro do stent a ser implantado. Foi escolhido um stent Vision $^{\text {TM }}$ 3,5/28 mm (Abbott Vascular, Temecula, Estados Unidos), posicionado no segmento a ser tratado
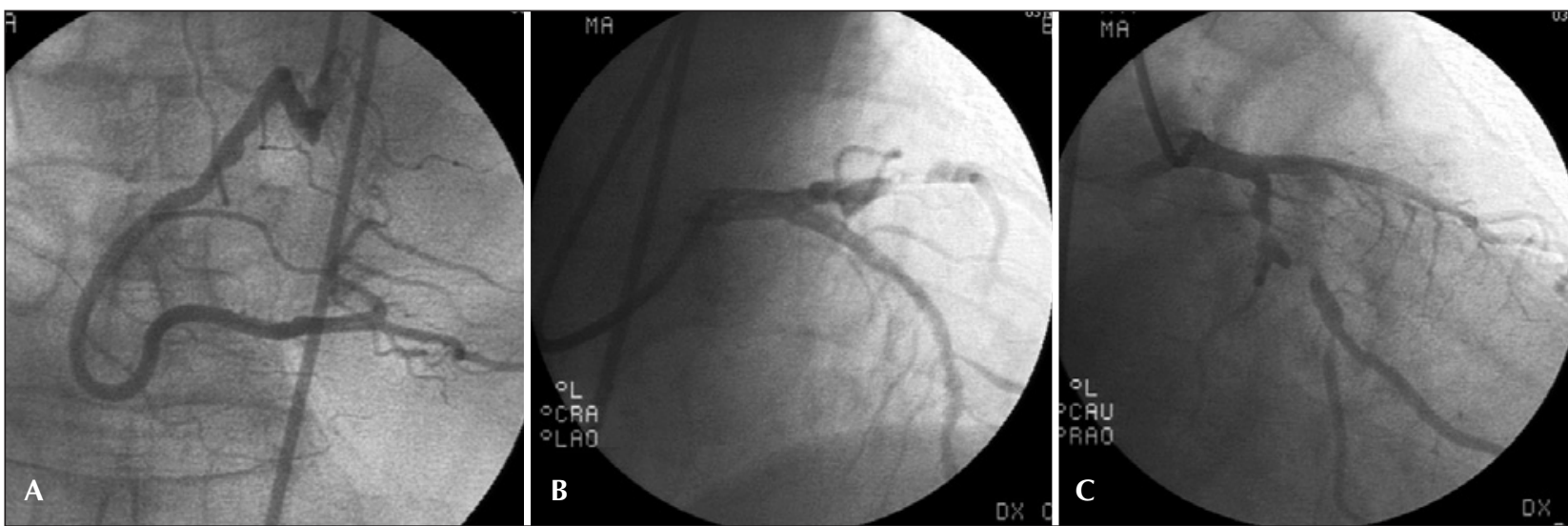

Figura 1 - Cinecoronariografia. Em A, coronária direita sem lesões obstrutivas significativas. Em B, tronco de coronária esquerda e artéria descendente anterior sem lesões obstrutivas significativas. Em C, artéria circunflexa com lesão obstrutiva de 30\% no óstio, lesão de $70 \%$ proximal seguida por outra de $90 \%$ no primeiro ramo marginal, o qual exibe lesão de $60 \%$ no óstio de um sub-ramo. 
com injeção de $0,5 \mathrm{ml}$ de meio de contraste. $O$ stent foi liberado com pressão de 5 atm, evitando dissecção de bordas (Figura 2). Em seguida, foi posicionado, com precisão milimétrica, balão não-complacente TREK NC ${ }^{\mathrm{TM}}$ 3,5/15 $\mathrm{mm}$ para a pós-dilatação, $1 \mathrm{~mm}$ aquém das bordas do stent, com a ajuda do software IC Stent ${ }^{\mathrm{TM}}$ (Siemens, Munique, Alemanha) e hiperinsuflado até 25 atm. O ultrassom intracoronário foi realizado novamente para documentação do implante ótimo do stent. Ao final, a angiografia de controle para a constatação do fluxo TIMI 3 foi realizada com 1,5 $\mathrm{ml}$, totalizando $3 \mathrm{ml}$ de contraste no procedimento (Figura 3).

\section{DISCUSSÃO}

Há poucos relatos na literatura utilizando-se a fluoroscopia, o ultrassom intracoronário ou outros métodos para guiar uma intervenção, visando a reduzir o volume de contraste ou, até mesmo, não utilizá-lo. Cook e Rees ${ }^{9}$ realizaram angioplastia na bifurcação aórtica em paciente com reação grave prévia ao contraste, utilizando ultrassom intracoronário e fluoroscopia para guiar o procedimento. Matthews e Thomas ${ }^{10}$ realizaram angioplastia com stent em veia central guiada por ultrassom intracoronário, sem o uso de contraste, obtendo resultado ótimo, confirmado pela expansão e pela aposição apropriadas do stent. Okura et al. ${ }^{11}$ submeteram paciente com alergia ao contraste a ICP com stent, escolhendo o tamanho da prótese com auxílio do ultrassom intracoronário, que serviu também para averiguar a expansão da prótese e a ausência de dissecção/aposição incompleta das hastes. Utilizaram também o fio-guia Doppler para constatar o fluxo anterógrado. Yamamoto et al. ${ }^{12}$ realizaram ICP em 2 pacientes com insuficiência renal crônica utilizando menos de $20 \mathrm{ml}$ de contraste.

Alternativas ao uso dos radiocontrastes iodados em intervenções endovasculares têm sido descritas. O gadolínio é proposto como uma das opções naqueles
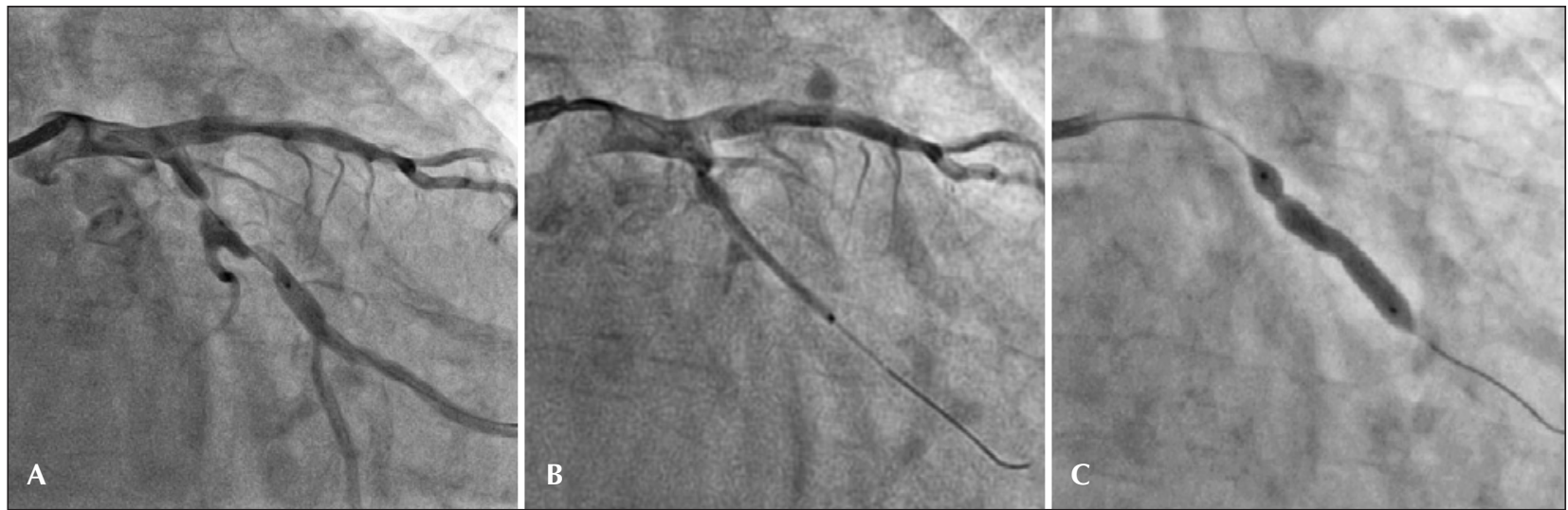

Figura 2 - Angioplastia: implante do stent. Em A, controle angiográfico após a pré-dilatação, com $1 \mathrm{ml}$ de contraste. Em B, posicionamento preciso do stent Vision ${ }^{\mathrm{MM}}$ 3,5/28 $\mathrm{mm}$ no segmento a ser tratado, com injeção de $0,5 \mathrm{ml}$ de contraste. Em C, implante do stent com pressão de 5 atm, evitando dissecção de bordas, observando-se expansão incompleta proximal do stent.

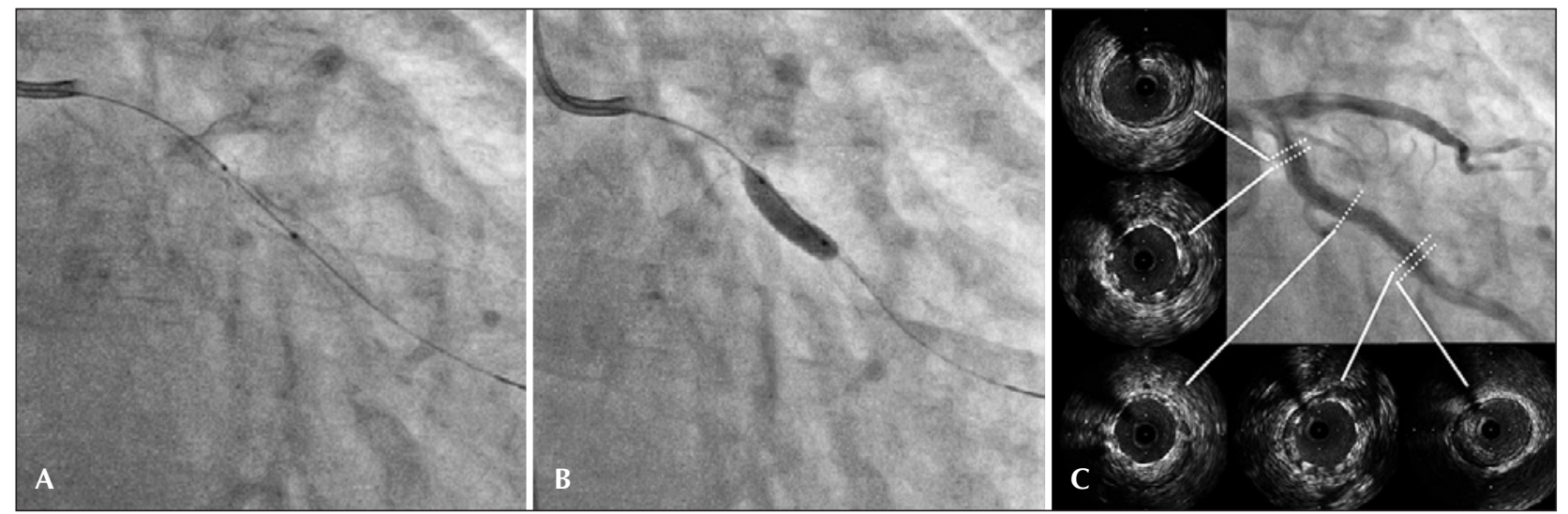

Figura 3 - Angioplastia: pós-dilatação e ultrassom intracoronário. Em A, posicionamento preciso do balão não-complacente TREK NC ${ }^{T M}$ 3,5/ $15 \mathrm{~mm}$ para a pós-dilatação, $1 \mathrm{~mm}$ aquém das bordas dos stents utilizando o software IC Stent ${ }^{\mathrm{TM}}$. Em B, hiperinsuflação a 25 atm, intrastent. Em C, angiografia de controle final, com 1,5 ml de contraste, totalizando $3 \mathrm{ml}$ de contraste para todo o procedimento. As imagens do ultrassom intracoronário documentam o implante ótimo do stent. 
pacientes com insuficiência renal ou outra razão para evitar-se o uso de contraste. ${ }^{13}$ A qualidade da imagem é adequada, ainda que inferior à dos contrastes iodados. Porém, não se pode esquecer o risco de desenvolvimento de fibrose sistêmica nefrogênica com esse agente, principalmente naqueles pacientes com doença renal em estágio avançado e sob diálise. Outra opção é o dióxido de carbono $\left(\mathrm{CO}_{2}\right)$. Caridi et al. ${ }^{14}$ descreveram angioplastias renais em 21 pacientes de alto risco usando o $\mathrm{CO}_{2}$ como meio de contraste durante angiografia por subtração digital.

Para aqueles com ampla experiência em ICP, a técnica de angioplastia coronária com mínima quantidade de contraste iodado é exequível e segura. O uso do ultrassom intracoronário auxilia no procedimento, mas não é obrigatório quando se usa pequena quantidade de contraste, como no caso descrito. A injeção de contraste é útil para checagem do fluxo coronário após a pré-dilatação (quando essa for realizada, ou seja, não necessária no implante de stent direto), no posicionamento preciso do stent e para verificação da qualidade do fluxo final. Para a realização de angioplastia totalmente sem o uso de meio de contraste, o ultrassom intracoronário é imprescindível, do mesmo modo que o fio-guia Doppler, para avaliação do fluxo anterógrado final. A qualidade adequada da imagem da fluoroscopia também facilita a intervenção, assim como ferramentas existentes nos equipamentos mais modernos de angiografia digital, como o software IC Stent ${ }^{\mathrm{TM}}$, usado nesse caso, que permite visibilização ótima das hastes do stent implantado.

Concluímos que diante do aumento significativo do número de exames com aplicação de agentes de contraste à base de iodo é necessária uma cooperação estreita entre o médico clínico e o intervencionista (cardiologista, radiologista ou vascular). Para a proteção dos pacientes é indispensável indicar os exames que empregam contraste iodado de forma responsável, identificar aqueles com fatores de risco, adotar medidas preventivas adequadas e monitorar a função renal. Ante equipamentos apropriados, operadores treinados e, sobretudo, bom-senso, cada vez mais novos desafios são transpostos na cardiologia intervencionista.

\section{CONFLITO DE INTERESSES}

Os autores declaram não haver conflito de interesses relacionado a este manuscrito.

\section{REFERÊNCIAS}

1. Maliborski A, Zukowski P, Nowicki G, Boguslawska R. Contrastinduced nephropathy - a review of current literature and guidelines. Med Sci Monit. 2011;17(9):RA199-204.

2. de Bie MK, van Rees JB, Herzog CA, Rabelink TJ, Schalij MJ, Jukema JW. How to reduce the incidence of contrast induced acute kidney injury after cardiac invasive procedures, a review and practical recommendations. Curr Med Res Opin. 2011; 27(7):1347-57.

3. Lepanto L, Tang A, Murphy-Lavallee J, Billiard JS. The Canadian Association of Radiologists Guidelines for the Prevention of Contrast-induced Nephropathy: A Critical Appraisal. Can Assoc Radiol J. 2011;62(4):238-42.

4. Pannu N, Wiebe N, Tonelli M; Alberta Kidney Disease Network. Prophylaxis strategies for contrast-induced nephropathy. JAMA. 2006;295(23):2765-79.

5. Tan N, Liu Y, Zhou YL, He PC, Yang JQ, Luo JF, et al. Contrast medium volume to creatinine clearance ratio: a predictor of contrast-induced nephropathy in the first 72 hours following percutaneous coronary intervention. Catheter Cardiovasc Interv. 2011;79(1):70-5.

6. Goldstein JA, Kern M, Wilson R. A novel automated injection system for angiography. J Interv Cardiol. 2001;14(2):147-52.

7. Anne G, Gruberg L, Huber A, Nikolsky E, Grenadier E, Boulus $M$, et al. Traditional versus automated injection contrast system in diagnostic and percutaneous coronary interventional procedures: comparison of the contrast volume delivered. J Invasive Cardiol. 2004;16(7):360-2.

8. Brosh D, Assali A, Vaknin-Assa H, Fuchs S, Teplitsky I, Shor $\mathrm{N}$, et al. The ACIST power injection system reduces the amount of contrast media delivered to the patient, as well as fluoroscopy time, during diagnostic and interventional cardiac procedures. Int J Cardiovasc Intervent. 2005;7(4):183-7.

9. Cook C, Rees M. Ultrasound and fluoroscopic-guided angioplasty over the aortic bifurcation in a patient with previous severe reaction to contrast medium. J Endovasc Ther. 2001;8(6): 648-51.

10. Matthews R, Thomas J. Intravascular ultrasound-guided central vein angioplasty and stenting without the use of radiographic contrast agents. J Clin Ultrassound. 2008;36(4):254-6.

11. Okura H, Nezuo S, Yoshida K. Successful stent implantation guided by intravascular ultrasound and a Doppler guidewire without contrast injection in a patient with allergy to iodinated contrast media. J Invsive Cardiol. 2011;23(7):297-99.

12. Yamamoto $E$, Takano $H$, Takayama M. Percutaneous coronary intervention under the rigid restriction of contrast media dose in patients with chronic renal insufficiency. J Invasive Cardiol. 2006;18(6):E169-72.

13. Nyman U, Golman K. Regarding "safety of gadolinium contrast angiography in patients with chronic renal insufficiency". J Vasc Surg. 2004;40(1):204; author reply 204.

14. Caridi JG, Stavropoulos SW, Hawkins IF Jr. CO2 digital subtraction angiography for renal artery angioplasty in high-risk patients. AJR Am J Roentgenol. 1999;173(6):1551-6. 